\title{
Doenças crônicas não transmissíveis e os fatores de risco em mulheres de Guaporé (RS)
}

\section{Chronic non-communicable diseases and risk factors in women of Guaporé (RS)}

\author{
Rafaela Romana, Josiane Sivierob \\ a Nutricionista. Pós-graduanda em Dietototerapia nos Ciclos da Vida com ênfase em Nutrição Clínica. Nutricionista da Associação Hospitalar \\ Manoel Francisco Guerreiro (AHMFG), Guaporé, RS \\ b Nutricionista. Doutora em Ciências da Saúde pela Pontifícia Universidade Católica do Rio Grande do Sul (PUCRS) com ênfase em Geriatria e Gerontologia. \\ Mestre em Gerontologia Biomédica (IGG, PUCRS). Docente do Curso de Nutrição da Faculdade Cenecista de Bento Gonçalves (CNEC) e da \\ Universidade de Caxias do Sul (UCS).
}

RESUMO

Objetivo: Avaliar a prevalência de doenças crônicas não transmissíveis e sua associação com fatores de risco em mulheres de Guaporé (RS).

Materiais e Métodos: Estudo transversal, realizado na microárea 23, durante os meses de maio e junho de 2016, com 165 mulheres. Dados coletados referentes à prevalência de doenças crônicas não transmissíveis (Diabetes Mellitus, Hipertensão Arterial e Dislipidemias) e medicamentação autorreferida, tabagismo, consumo de bebidas alcoólicas, atividade física, índice de massa corporal, circunferência da cintura e consumo alimentar. A análise estatística foi realizada através do programa Statistical Package for the Social Sciences. Os testes foram t-student para as médias, teste qui-quadrado de Pearson para associações e teste qui-quadrado para variáveis ordinais, sendo o nível de significância de $5 \%$.

Resultados: Avaliaram-se 165 mulheres, das quais 29,1\% possuíam $\geq 60$ anos, 69,7\% eram casadas ou moravam com companheiro, 44,8\% possuíam $\geq 13$ anos de escolaridade, 83,6\% possuíam renda de 1-2 salários mínimos. Verificou-se que o índice de massa corporal foi $27,8 \pm 5,6 \mathrm{~kg} / \mathrm{m}^{2}$, encontrando-se em sobrepeso. A circunferência da cintura foi $98,9 \pm 13,9 \mathrm{~cm}$, classificando-se em risco. Ao associar os fatores de risco consumo alimentar, atividade física, tabagismo, bebidas alcóolicas, não se encontrou associação significativa. No entanto encontrou-se maior prevalência de doenças crônicas não transmissíveis em mulheres com maior faixa etária, de menor escolaridade e renda, viúvas, com circunferência da cintura elevada e índice de massa corporal de sobrepeso. Constatou-se a presença de doenças crônicas não transmissíveis em 38,2\% das mulheres.

Conclusão: A prevalência de doenças crônicas não transmissíveis associou-se com alguns dos fatores de risco estudados, desses o estado civil entre as viúvas, menor escolaridade, menor renda, com baixa ingestão de álcool e risco mais elevado para complicações metabólicas, conforme circunferência da cintura.

Palavras-chave: doenças crônicas; prevalência; consumo alimentar; fatores de risco. 


\section{INTRODUÇÃO}

Doenças crônicas não transmissíveis (DCNT) são doenças multifatoriais que se desenvolvem ao longo de nossas vidas ${ }^{1}$ e constituem atualmente a maior causa de morbimortalidade do mundo. Os principais fatores que causam essas doenças incluem fatores de risco modificáveis, como tabagismo, consumo nocivo de bebida alcoólica, inatividade física e alimentação inadequada² ${ }^{2}$

Segundo estimativas, aproximadamente $80 \%$ das mortes ocorrem em países de baixa e média renda, e um terço em pessoas com idade superior a 60 anos. No Brasil são responsáveis por $72 \%$ das causas de mortes, com destaque para as doenças cardiovasculares, diabetes, neoplasias e doenças respiratórias crônicas ${ }^{2}$. Em outros países, como no México, as doenças crônicas são problemas de saúde urgente, englobando as doenças cardíacas coronarianas, diabetes e acidente vascular cerebral ${ }^{3}$.

Conforme a Organização Pan Americana da Saúde, os fatores de risco relativos às DCNT são semelhantes em todos os países. Atualmente o tabagismo, os alimentos com altas concentrações de gorduras trans e saturadas, o sal e o açúcar em excesso, especialmente em bebidas adoçadas, o sedentarismo, bem como o consumo excessivo de álcool, causam mais de dois terços de todos os novos casos de DCNT e aumentam o risco de complicações em pessoas que já sofrem dessas doenças ${ }^{4}$.

Os dados da Vigilância de Doenças Crônicas por Inquérito Telefônico (VIGITEL) ${ }^{5}$ mostraram que, nos 26 estados estudados mais o Distrito Federal, o percentual de diagnóstico médico prévio de hipertensão arterial foi de $24,8 \%$, sendo maior em mulheres $(26,8 \%)$ do que em homens (22,5\%), e o diagnóstico médico prévio de diabetes foi de $8,0 \%$, sendo de $7,3 \%$ entre os homens e $8,7 \%$ entre as mulheres. Para as dislipidemias, o diagnóstico foi de $20,0 \%$, sendo maior em mulheres $(22,2 \%)$ do que nos homens $(17,6 \%)^{5}$.

Além disso, foi observado na Pesquisa Nacional de Saúde ${ }^{6}$ que o percentual de indivíduos que consumiam cinco porções diárias de frutas e hortaliças foi de $37,3 \%$, o consumo de feijão foi referido por $71,9 \%$, o consumo de carne ou frango com excesso de gordura foi de $37,2 \%$, relataram que bebem leite integral $60,6 \%$ dos indivíduos, e o consumo de doces como bolos, tortas, chocolates foi mencionado por $21,7 \%$.

Com base no exposto acima, torna-se cada vez mais importante identificar a presença de comportamentos relacionados com um estilo de vida não saudável, atribuídos ao sedentarismo, tabagismo, consumo de álcool, estado nutricional e práticas alimentares inadequadas. $\mathrm{O}$ objetivo do presente estudo foi avaliar a prevalência de doenças crônicas não transmissíveis e sua associação com fatores de risco em mulheres de Guaporé-RS.

\section{MATERIAIS E MÉTODOS}

Trata-se de um estudo transversal realizado por meio de entrevistas estruturadas com mulheres adultas e idosas da microárea 23, da área urbana, do município de Guaporé/RS. O presente estudo foi concentrado nesta área da ESF do Bom Jesus, por abranger a maior parte da população do bairro, sendo de 152 famílias, compostas por 184 indivíduos do sexo feminino. O município é composto por seis Estratégias de Saúde da Família (ESF), divididas em microáreas específicas, cuja subdivisão foi realizada pelas ESF. A partir disso a obtenção da amostra foi por conveniência.

Todas as moradoras da microárea 23 foram convidadas para participar da pesquisa por meio de visitas domiciliares juntamente ao Agente Comunitário de Saúde. Das 184 mulheres estudadas, apenas 165 fizeram parte da amostra, atendendo aos critérios de inclusão, que foram: mulheres adultas ou idosas, moradoras da microárea 23, lúcidas e alfabetizadas. Foram excluídas 19 mulheres, devido aos critérios de exclusão, que foram: gestantes, com dificuldades auditivas ou incapacitadas de se comunicar, devido à mudez, e também mulheres que se encontravam no cadastro, mas que se mudaram da microárea durante a aplicação da pesquisa.

A coleta de dados ocorreu nos meses de maio de 2016 a junho de 2016. Foi aplicado um questionário estruturado com dados referentes às características demográficas (idade e estado civil) socioeconômicas (escolaridade, ocupação profissional e renda) e comportamentais (atividade física, tabagismo, bebidas alcoólicas e consumo alimentar). As doenças crônicas não transmissíveis (Diabetes Mellitus, Hipertensão Arterial e Dislipidemias - nesse caso, Colesterol e Triglicerídeos $)^{5}$ e o uso de medicamentos para o tratamento das patologias foram autorreferidas pelas entrevistadas. Os dados de massa corporal, estatura e circunferência da cintura foram aferidos.

O nível de atividade física foi avaliado através do International Physical Activity Questionnaire (IPAQ) ${ }^{7}$, em sua versão curta, a qual classifica como insuficientemente ativos aqueles indivíduos com escore de atividade física $<150$ minutos/semana. No que se refere ao comportamento tabagista, foi considerado fumante o indivíduo que fuma um ou mais cigarros por dia há mais de um mês, e ex-fumante aquele que não fuma há mais de um mês. Foram considerados para o consumo abusivo de bebidas alcoólicas ingestão maior que quatro doses, respectivamente em uma única ocasião em pelo menos uma vez nos últimos 30 dias. Uma dose de 
bebida alcoólica corresponde a uma lata de cerveja, a uma taça de vinho ou a uma dose de cachaça, whisky ou qualquer outra bebida alcoólica destilada, conforme estudo realizado por ROCHA et al. ${ }^{8}$

Para avaliar alimentação, foi aplicado o Questionário de avaliação do consumo alimentar adaptado do Estudo Longitudinal de Saúde do Adulto (ELSA Brasil) ${ }^{12}$. Trata-se de um instrumento quantitativo que, após adequação, totalizou em 10 grupos alimentares, considerando os determinados alimentos: Grupo dos Pães, Cereais e Tubérculos (arroz branco, batata inglesa ou doce, mandioca, aveia, granola ou outros cereais, pão integral, pão de centeio, pão francês, pão de forma e pão caseiro, bolo simples, biscoito salgado e biscoito doce); Grupo das Frutas ou Salada de Frutas (laranja, banana, maçã, manga, entre outras); Grupo dos Folhosos (alface, chicória, rúcula, espinafre, entre outros); Grupo dos Legumes e Verduras Cozidas (repolho, tomate, abóbora, vagem, cenoura, beterraba, couve-flor, brócolis, entre outros); Grupo das Leguminosas (feijão preto, vermelho, de cor, lentilha, grão de bico e ervilha); Grupo das Oleaginosas (nozes, castanha-de-caju, castanha-do-pará, amendoim e amêndoas); Grupo das Carnes, Leite e Derivados (leite desnatado, semidesnatado, leite integral, iogurte normal, iogurte light, queijos amarelos, colonial, minas padrão, mozarela, prato, cheddar, carne de boi sem osso, bife, carne moída, carne ensopada, carne de porco, linguiça, salsichão, presunto, mortadela, copa ou salame, peito de frango, chester, peru, frango cozido ou outras partes, peixe cozido, assado, ensopado, grelhado ou frito); Grupo das Massas e outras preparações (pizza, salgados assados, macarrão, lasanha, tortéi, caneloni e ravióli); Grupo dos Doces (chocolate em barra, bombom, brigadeiro, negrinho, doce de festa e doce de leite); Grupo das Bebidas (refrigerante, café preto, suco natural e suco industrializado). Para esses alimentos, foi analisado se o consumo era praticado diariamente, semanalmente, mensalmente e se não havia consumo. A aplicação das entrevistas, englobando todos os questionamentos, durou aproximadamente de 20 a 40 minutos.

Foram verificados dados antropométricos, como peso $(\mathrm{kg})$ e estatura $(\mathrm{m})$. A massa corporal foi obtida com a balança digital marca Plenna ${ }^{\circledR}$, com capacidade de $150 \mathrm{~kg}$ e precisão de $100 \mathrm{~g}$, utilizando o procedimento padrão ${ }^{9}$. A estatura foi obtida com o estadiômetro portátil da marca SECA ${ }^{\circledR}$, modelo 208, com capacidade de $200 \mathrm{~cm}$ e precisão de $0,1 \mathrm{~cm}$ fixado com fita adesiva em uma parede lisa, sem rodapé, utilizando o procedimento padrão ${ }^{9}$. A Circunferência da cintura foi

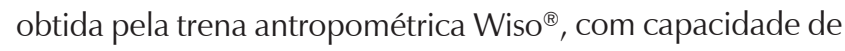
$2 \mathrm{~m}$, utilizando o procedimento padrão ${ }^{10}$. Para a classificação do estado nutricional segundo Índice de Massa Corporal (IMC), foram utilizados os pontos de corte preconizados pelo
World Health Organization $1995^{8}$ para IMC adultos, World Health Organization $2000^{10}$ para circunferência da Cintura (CC) e The Nutrition Screening Initiative ${ }^{11}$ para IMC idosos.

A obtenção das informações ocorreu somente após aprovação do Comitê de Ética em Pesquisa da Faculdade Cenecista de Bento Gonçalves (CNEC), sob o registro número1.497.666. Este estudo seguiu as recomendações da Resolução 466/12 do Conselho Nacional de Saúde.

Após a coleta, os dados foram digitados em programa Microsoft Office Excell ${ }^{\circledR}$ 2013, a fim de codificar as respostas. Posteriormente os dados foram analisados através do programa Statistical Package for the Social Sciences (SPSS ${ }^{\circledR}$ ) versão 21.0. As variáveis quantitativas foram descritas por média e desvio padrão ou mediana e amplitude interquartílica. As variáveis categóricas foram descritas por frequências absolutas e relativas. Para comparar médias, o teste t-student foi aplicado. E para avaliar a associação entre os dados demográficos, de estilo de vida, estado nutricional e consumo alimentar com a prevalência de doenças crônicas não transmissíveis (DCNT), o teste qui-quadrado de Pearson foi aplicado. Para as variáveis ordinais, o teste qui-quadrado de tendência linear foi utilizado. O nível de significância adotado foi de $5 \%(p<0,05)$.

\section{RESULTADOS}

Do total de 165 mulheres avaliadas no presente estudo, a média de idade foi $49,5 \pm 17,2$ anos, sendo que a maioria (29,1\%) possuía $\geq 60$ anos, 69,7\% eram casadas ou moravam com um companheiro, 44,8\% possuíam 13 anos ou mais de escolaridade. No que se refere à ocupação profissional relatada, a maioria $(29,1 \%)$ possuíam outra profissão não se enquadrando entre as questionadas e citadas na tabela, e $83,6 \%$ informou ter renda entre 1-2 salários mínimos

\section{(Tabela 1).}

Ao analisar a prevalência das doenças crônicas não transmissíveis relatadas pelas mulheres, constatou-se que $29,1 \%$ eram hipertensas, seguido de $20,6 \%$ que possuíam colesterol elevado. Nos demais fatores investigados, apenas $4,2 \%$ eram diabéticas e 5,5\% possuíam triglicerídeos elevados. A presença de no mínimo uma das doenças crônicas não transmissíveis (hipertensão, diabetes melitus, colesterol e triglicerídeos elevados) foi relatada por $38,2 \%$ das mulheres.

O uso de medicação contínua para o tratamento das doenças crônicas autorreferido foi caracterizado em 38,2\% das mulheres. No que se refere à atividade física, mais da metade da amostra $(65,5 \%)$ era sedentária. Sobre a ingestão de álcool, sendo mais de quatro doses nos últimos 30 dias, apenas 5,5\% relataram consumir. Quanto ao tabagismo atual, somente 3,6\% relataram realizar essa prática (Tabela 1). 
Tabela 1. Caracterização da amostra, segundo dados demográficos, socioeconômicos e comportamentais em mulheres de Guaporé.

\begin{tabular}{|c|c|}
\hline Variáveis & $n=165$ \\
\hline Idade (anos) - média \pm DP & $49,5 \pm 17,2$ \\
\hline \multicolumn{2}{|l|}{ Faixa etária - n (\%) } \\
\hline 20 a 29 anos & $22(13,3)$ \\
\hline 30 a 39 anos & $31(18,8)$ \\
\hline 40 a 49 anos & $34(20,6)$ \\
\hline 50 a 59 anos & $30(18,2)$ \\
\hline$\geq 60$ anos & $48(29,1)$ \\
\hline \multicolumn{2}{|l|}{ Estado civil - n (\%) } \\
\hline Solteira & $21(12,7)$ \\
\hline Casada / mora com um (a) companheiro (a) & $115(69,7)$ \\
\hline Separada / divorciada / desquitada & $9(5,5)$ \\
\hline Viúva & $20(12,1)$ \\
\hline Escolaridade (anos) - md (P25-P75) & $8(5-13)$ \\
\hline \multicolumn{2}{|l|}{ Nível de escolaridade - n (\%) } \\
\hline $0-4$ & $21(12,7)$ \\
\hline $5-8$ & $60(36,4)$ \\
\hline $9-12$ & $10(6,1)$ \\
\hline 13 ou mais & $74(44,8)$ \\
\hline \multicolumn{2}{|l|}{ Ocupação profissional - n (\%) } \\
\hline Agricultura (campo, fazenda ou com pesca) & $6(3,6)$ \\
\hline Comércio, banco, transporte, hotelaria ou outros serviços & $13(7,9)$ \\
\hline Funcionária do governo federal, estadual ou municipal & $17(10,3)$ \\
\hline Profissional liberal, professora ou técnica de nível superior & $3(1,8)$ \\
\hline Atividades informais & $3(1,8)$ \\
\hline Doméstica & $2(1,2)$ \\
\hline Do lar (sem remuneração) & $15(9,1)$ \\
\hline Outras ocupações profissionais & $48(29,1)$ \\
\hline Sem ocupação profissional & $1(0,6)$ \\
\hline Aposentada e/ou pensionista & $47(28,5)$ \\
\hline Renda mensal (reais) - md (P25-P75) & $\begin{array}{c}1000 \\
(880-1760)\end{array}$ \\
\hline \multicolumn{2}{|l|}{ Faixas de renda $-\mathrm{n}(\%)$} \\
\hline Até 1 salário mínimo $(\mathrm{R} \$ 880,00)$ & $68(41,2)$ \\
\hline De 1,1 até 2 salários mínimos & $70(42,4)$ \\
\hline De 2,1 até 5 salários mínimos & $10(6,1)$ \\
\hline Mais de 5 salários mínimos & $1(0,6)$ \\
\hline Nenhuma renda & $16(9,7)$ \\
\hline Uso de medicação contínua - n (\%) & $63(38,2)$ \\
\hline \multicolumn{2}{|l|}{ Atividade física - n (\%) } \\
\hline Muito ativo & $15(9,1)$ \\
\hline Ativo & $33(20,0)$ \\
\hline Irregularmente ativo & $9(5,5)$ \\
\hline Sedentário & $108(65,5)$ \\
\hline Ingestão de álcool >4 doses nos últimos 30 dias $-\mathrm{n}(\%)$ & $9(5,5)$ \\
\hline Tabagismo atual - n (\%) & $6(3,6)$ \\
\hline Tabagismo no passado - n (\%) & $23(13,9)$ \\
\hline
\end{tabular}

DP: desvio padrão; $p$ : significância; n: número; \%: porcentagem; md: mediana.
Com relação à avaliação do estado nutricional, verificou-se que maioria se encontrava em sobrepeso $(41,8 \%)$, seguido de 20,6\% em obesidade. A CC média foi de $98,9 \pm 13,9 \mathrm{~cm}$, no qual, ao distribuir as classificações, verificou-se que $17,6 \%$ e $75,2 \%$ apontaram risco elevado e muito elevado para complicações metabólicas, respectivamente (Tabela 2).

Na Tabela 3, encontra-se o consumo alimentar obtido através do questionário de frequência alimentar. O consumo dos alimentos provenientes do grupo dos pães, cereais e tubérculos teve maior destaque para o consumo semanal $(94,5 \%)$ para arroz, batata inglesa ou doce e mandioca, e $61,2 \%$ relataram não consumir cereais, pão integral ou pão de centeio.

Para os alimentos do grupo das frutas, vegetais, oleaginosas e leguminosas, o consumo foi de $73,9 \%$ para ingestão diária de frutas frescas e salada de frutas, consumo semanal de leguminosas $(77,0 \%)$, de $76,4 \%$ para legumes e verduras cozidas, e não consumo de $43,6 \%$ para o grupo das oleaginosas.

Quanto aos alimentos do grupo alimentar das carnes, leites e derivados, destacou-se o não consumo de leite desnatado e semidesnatado (83,0\%) e $92,7 \%$ de não consumo iogurte light. Para o consumo de queijos amarelos, $41,8 \%$ relataram consumo semanal. As carnes foram ingeridas semanalmente, tendo maior destaque o consumo de carne de boi sem osso, bife, carne moída, carne ensopada $(93,9 \%)$, assim como peito de frango, chester, peru, frango cozido ou outras preparações (93,9\%).

Tabela 2. Avaliação antropométrica e classificação do estado nutricional em mulheres de Guaporé.

\begin{tabular}{|c|c|}
\hline Variáveis & $n=165$ \\
\hline Peso $(\mathrm{kg})$ - média $\pm \mathrm{DP}$ & $69,7 \pm 14,9$ \\
\hline Altura $(m)$ - média \pm DP & $1,58 \pm 0,07$ \\
\hline IMC $\left(\mathrm{kg} / \mathrm{m}^{2}\right)-$ média $\pm \mathrm{DP}$ & $27,8 \pm 5,6$ \\
\hline \multicolumn{2}{|l|}{ Classificação do IMC - n (\%) } \\
\hline Baixo peso & $6(3,6)$ \\
\hline Eutrofia & $56(33,9)$ \\
\hline Sobrepeso & $69(41,8)$ \\
\hline Obesidade & $34(20,6)$ \\
\hline CC $(\mathrm{cm})-$ média $\pm \mathrm{DP}$ & $98,9 \pm 13,9$ \\
\hline \multicolumn{2}{|l|}{ Classificação da CC - n (\%) } \\
\hline Sem risco & $12(7,3)$ \\
\hline Risco elevado para complicações metabólicas & $29(17,6)$ \\
\hline Risco muito elevado para complicações metabólicas & $124(75,2)$ \\
\hline
\end{tabular}


Tabela 3. Consumo alimentar de mulheres de Guaporé.

\begin{tabular}{|c|c|c|c|c|}
\hline Alimentos & $\begin{array}{l}\text { Diário } \\
\text { n (\%) }\end{array}$ & $\begin{array}{c}\text { Semanal } \\
\text { n (\%) }\end{array}$ & $\begin{array}{c}\text { Mensal } \\
\text { n (\%) }\end{array}$ & $\begin{array}{c}\text { Não consome } \\
\mathbf{n}(\%)\end{array}$ \\
\hline \multicolumn{5}{|l|}{ Grupo dos Pães, Cereais e Tubérculos } \\
\hline Arroz, batata inglesa ou doce e mandioca & $2(1,2)$ & $156(94,5)$ & $7(4,2)$ & $0(0,0)$ \\
\hline Cereais, pão integral ou pão de centeio & $20(12,1)$ & $40(24,2)$ & $4(2,4)$ & $101(61,2)$ \\
\hline Pão francês, forma ou caseiro & $93(56,4)$ & $46(27,9)$ & $0(0,0)$ & $26(15,8)$ \\
\hline Bolos, biscoito doce ou salgado & $8(4,8)$ & $92(55,8)$ & $20(12,1)$ & $45(27,3)$ \\
\hline \multicolumn{5}{|l|}{ Grupo das Frutas ou Salada de Fruta } \\
\hline Laranja, banana, mamão, maçã, manga, entre outras & $122(73,9)$ & $39(23,6)$ & $1(0,6)$ & $3(1,8)$ \\
\hline \multicolumn{5}{|l|}{ Grupo dos Folhosos } \\
\hline Alface, chicória, rúcula, espinafre, entre outros. & $97(58,8)$ & $52(31,5)$ & $0(0,0)$ & $16(9,7)$ \\
\hline \multicolumn{5}{|l|}{ Grupo dos Legumes e Verduras Cozidas } \\
\hline Repolho, tomate, abóbora, vagem, cenoura, beterraba, couve-flor, brócolis, entre outros & $30(18,2)$ & $126(76,4)$ & $0(0,0)$ & $9(5,5)$ \\
\hline \multicolumn{5}{|l|}{ Grupo das Leguminosas } \\
\hline Feijão preto, vermelho, branco ou de cor, lentilha, grão de bico, ervilha & $27(16,4)$ & $127(77,0)$ & $4(2,4)$ & $7(4,2)$ \\
\hline \multicolumn{5}{|l|}{ Grupo das Oleaginosas } \\
\hline Nozes, castanha de caju ou Pará, amendoim ou amêndoas & $11(6,7)$ & $56(33,9)$ & $26(15,8)$ & $72(43,6)$ \\
\hline \multicolumn{5}{|l|}{ Grupo das Carnes, Leites e Derivados } \\
\hline Leite desnatado ou Semi-desnatado & $22(13,3)$ & $6(3,6)$ & $0(0,0)$ & $137(83,0)$ \\
\hline Leite integral & $59(35,8)$ & $15(9,1)$ & $2(1,2)$ & $89(53,9)$ \\
\hline logurte normal & $16(9,7)$ & $43(26,1)$ & $12(7,3)$ & $94(57,0)$ \\
\hline logurte light & $5(3,0)$ & $6(3,6)$ & $1(0,6)$ & $153(92,7)$ \\
\hline Queijos amarelos, colonial, minas, padrão, mussarela, prato, cheddar entre outros & $64(38,8)$ & $69(41,8)$ & $2(1,2)$ & $30(18,2)$ \\
\hline Carnes de boi sem osso, bife, carne moída ou carne ensopada & $3(1,8)$ & $155(93,9)$ & $5(3,0)$ & $2(1,2)$ \\
\hline Carnes de porco, linguiça, salsichão, presunto, mortadela, copa ou salame & $0(0,0)$ & $79(47,9)$ & $56(33,9)$ & $30(18,2)$ \\
\hline Peito de frango, chester, peru, frango cozido ou outras partes. & $2(1,2)$ & $155(93,9)$ & $4(2,4)$ & $4(2,4)$ \\
\hline Peixe cozido, assado, ensopado, grelhado ou frito. & $0(0,0)$ & $43(26,1)$ & $74(44,8)$ & $48(29,1)$ \\
\hline \multicolumn{5}{|l|}{ Grupo das Massa e Outras Preparações } \\
\hline Pizza, salgados assados, macarrão, caneloni, lasanha, ravióli ou tortéi. & $1(0,6)$ & $133(80,6)$ & $23(13,9)$ & $8(4,8)$ \\
\hline \multicolumn{5}{|l|}{ Grupo dos Doces } \\
\hline Chocolate em barra, bombom, negrinho, doce de leite ou doce de festa. & $12(7,3)$ & $54(32,7)$ & $42(25,5)$ & $57(34,5)$ \\
\hline \multicolumn{5}{|l|}{ Grupo das Bebidas } \\
\hline Refrigerante & $10(6,1)$ & $75(45,5)$ & $23(13,9)$ & $57(34,5)$ \\
\hline Café preto & $89(53,9)$ & $18(10,9)$ & $1(0,6)$ & $57(34,5)$ \\
\hline Suco natural & $28(17,0)$ & $62(37,6)$ & $2(1,2)$ & $73(44,2)$ \\
\hline Suco industrializado & $24(14,5)$ & $49(29,7)$ & $2(1,2)$ & $90(54,5)$ \\
\hline
\end{tabular}

n: número; \%: porcentagem.

No grupo das massas e outras preparações, o maior destaque foi semanalmente com $80,6 \%$ de consumo, e o grupo dos doces, que $34,5 \%$ não consomem. Entre o grupo das bebidas, destacam-se o consumo semanal de refrigerantes $(45,5 \%)$ e o consumo diário de café preto $(53,9 \%)$, assim como o não consumo de suco natural $(44,2 \%)$ e, por fim, $54,5 \%$ não consomem suco industrializado.

Conforme a Tabela 4, observam-se as associações entre dados demográficos, de estilo de vida e antropométricos com as DCNT, a partir das quais constata-se que quanto maior a faixa etária, maior a prevalência de DCNT $(p<0,001)$.
Além disso, observou-se que as maiores prevalências de DCNT foram encontradas em viúvas, com menor escolaridade, menor renda, com baixa ingestão de álcool e risco mais elevado para complicações metabólicas, conforme a circunferência da cintura. Não houve associação significativa da DCNT com atividade física $(p=0,569)$, tabagismo atual $(p=1,000)$ e passado $(p=0,896)$ e estado nutricional, pelo IMC $(p=0,099)$.

No entanto, se comparadas as médias de IMC entre as que apresentam DCNT e as que não apresentam, respectivamente, a diferença foi estatisticamente significativa, sendo o IMC 
Tabela 4. Associações dos dados demográficos, de estilo de vida e antropométricos com as DCNT em mulheres de Guaporé.

\begin{tabular}{|c|c|c|c|}
\hline Variáveis & $\mathbf{n}$ & $\begin{array}{l}\text { Prevalência } \\
\text { de DCNT }\end{array}$ & $p$ \\
\hline Faixa etária & & & $<0,001$ \\
\hline 20 a 29 anos & 22 & $4,5 \%$ & \\
\hline 30 a 39 anos & 31 & $9,7 \%$ & \\
\hline 40 a 49 anos & 34 & $17,6 \%$ & \\
\hline 50 a 59 anos & 30 & $56,7 \%$ & \\
\hline$\geq 60$ anos & 48 & $75,0 \%$ & \\
\hline Estado civil & & & 0,001 \\
\hline Solteira & 21 & $9,5 \%$ & \\
\hline $\begin{array}{l}\text { Casada / mora com um (a) } \\
\text { companheiro (a) }\end{array}$ & 115 & $36,5 \%$ & \\
\hline $\begin{array}{l}\text { Separada / divorciada / } \\
\text { desquitada }\end{array}$ & 9 & $55,6 \%$ & \\
\hline Viúva & 20 & $70,0 \%$ & \\
\hline Nível de escolaridade & & & $<0,001$ \\
\hline $0-4$ & 21 & $66,7 \%$ & \\
\hline $5-8$ & 60 & $56,7 \%$ & \\
\hline $9-12$ & 10 & $10,0 \%$ & \\
\hline 13 ou mais & 74 & $18,9 \%$ & \\
\hline Faixas de renda & & & 0,009 \\
\hline Até 1 salário mínimo & 68 & $54,4 \%$ & \\
\hline De 1,1 até 2 salários mínimos & 70 & $27,1 \%$ & \\
\hline De 2,1 até 5 salários mínimos & 10 & $20,0 \%$ & \\
\hline Mais de 5 salários mínimos & 1 & $0,0 \%$ & \\
\hline Nenhuma renda & 16 & $31,3 \%$ & \\
\hline Atividade física & & & 0,569 \\
\hline Muito ativo & 15 & $26,7 \%$ & \\
\hline Ativo & 33 & $39,4 \%$ & \\
\hline Irregularmente ativo & 9 & $55,6 \%$ & \\
\hline Sedentário & 108 & $38,0 \%$ & \\
\hline $\begin{array}{l}\text { Ingestão de álcool >4 doses nos } \\
\text { últimos } 30 \text { dias }\end{array}$ & & & 0,014 \\
\hline Sim & 9 & $0,0 \%$ & \\
\hline Não & 156 & $40,4 \%$ & \\
\hline Tabagismo atual & & & 1,000 \\
\hline Sim & 6 & $33,3 \%$ & \\
\hline Não & 159 & $38,4 \%$ & \\
\hline Tabagismo no passado & & & 0,896 \\
\hline Sim & 23 & $34,8 \%$ & \\
\hline Não & 142 & $38,7 \%$ & \\
\hline Classificação do IMC & & & 0,099 \\
\hline Baixo peso & 6 & $50,0 \%$ & \\
\hline Eutrofia & 56 & $26,8 \%$ & \\
\hline Sobrepeso & 69 & $47,8 \%$ & \\
\hline Obesidade & 34 & $35,3 \%$ & \\
\hline Classificação da CC & & & 0,017 \\
\hline Sem risco & 12 & $16,7 \%$ & \\
\hline Risco elevado & 29 & $20,7 \%$ & \\
\hline Risco muito elevado & 124 & $44,4 \%$ & \\
\hline
\end{tabular}

n: número; \%: porcentagem; IMC: índice de massa corporal; CC: circunferência da cintura; DCNT: doenças crônicas não transmissíveis; teste: teste qui-quadrado de Pearson. médio mais elevado nas mulheres que apresentam DCNT $(29,6 \pm 6,0)$ versus as que não apresentam DCNT $(26,7 \pm 5,1)$; $p=0,001$. Ao se analisar a relação entre prevalência das doenças crônicas não transmissíveis e consumo alimentar, não houve associação significativa.

\section{DISCUSSÃO}

A prevalência de doenças crônicas não transmissíveis encontrada no presente estudo foi de $38,2 \%$, resultado inferior ao encontrado por estudo realizado em uma população de 112 indivíduos com idade superior a 60 anos, em que $83,0 \%$ apresentaram pelo menos uma DCNT ${ }^{13}$. Outro estudo, no qual $77,5 \%$ da população eram mulheres, obteve estimativa de $44,8 \%{ }^{8}$. Ambos os estudos destacaram que a maioria das mulheres possuía mais de 60 anos.

Dentre todas as doenças crônicas não transmissíveis relatadas no presente estudo, destaca-se que $29,1 \%$ das mulheres eram hipertensas e 20,6\% possuíam colesterol elevado, ao contrário do estudo de Cavalcanti et al. ${ }^{14}$, que obteve resultados superiores, com $56,4 \%$ da população de hipertensos e 33,3\% possuíam alguma dislipidemia.

Além disso, observou-se prevalência de DCNT entre as mulheres viúvas, com menor escolaridade e menor renda. $\mathrm{O}$ estado civil, a renda e o nível de escolaridade podem estar interferindo no acesso aos cuidados com a saúde, no que diz respeito à forma do ser humano viver e às condições que ele tem para sobreviver ${ }^{15}$.

Outro fator que se destaca como contribuinte para a prevalência de doenças crônicas não transmissíveis são as variáveis do estado nutricional IMC e $\mathrm{CC}^{16}$. No presente estudo, houve risco elevado e muito elevado, conforme a CC, não havendo associação significativa com estado nutricional, pelo IMC, no entanto, em comparação com as médias, as diferenças foram significativas, sendo de IMC elevado para aquelas que apresentaram alguma DCNT. Conforme estudo de Scherer et al. ${ }^{15}$, houve associação significativa, com maior percentual de sobrepeso em $62,5 \%$ das mulheres através do IMC, se comparado entre os homens, $60,3 \%$. De acordo com outros achados, a variável da CC sempre se manteve com maior destaque nas mulheres, com valores respectivamente de $66,0 \%$ e $61,0 \%{ }^{17,18}$.

Estudo realizado em idosos, no qual 94,0\% da amostra apresentam mulheres com idades entre 60 a 69 anos, identificou, segundo classificação do IMC, 46,2\% da amostra com sobrepeso e 40,2\% obesidade grau I. Com relação à CC, $97,4 \%$ foram classificados como obesos abdominais com risco muito elevado para doenças cardiovasculares ${ }^{14}$. Em estudo realizado em coreanos, cerca de um terço tem índice de massa corporal superior a $25,0 \mathrm{~kg} / \mathrm{m}^{2}$, sendo aumento consideravelmente de $26 \%$ para $34 \%$ em mulheres ${ }^{15}$. 
A maioria das mulheres do presente estudo relatou ser sedentária $(65,5 \%)$, contudo não houve associação significativa com as DCNT no presente estudo. Esse é um achado semelhante ao encontrado por Scherer et al. ${ }^{17}$, no qual não se observou associação positiva com a prevalência de DCNT. No entanto destaca-se que, segundo dados da Pesquisa Nacional da Saúde ${ }^{20}$, a prevalência de inatividade física foi de 46,0\%, sendo significativamente maior entre as mulheres, existindo diferença entre as faixas etárias e prevalência maior de inatividade física entre os idosos.

Verificou-se que não houve associação significativa com tabagismo atual e passado, ao contrário do estudo Rocha et al. ${ }^{8}$, em que foi encontrada associação positiva, sendo que os entrevistados fumantes tiveram risco de desenvolvimento de DCNT respectivamente de $2,0(p<0,02)$ e 1,68 vezes $(p<0,03)$ maior que os não fumantes.

Quanto ao consumo abusivo de bebidas alcoólicas, sendo mais de quatro doses nos últimos 30 dias, apenas $5,5 \%$ relataram consumir. Houve maiores prevalências de DCNT naquelas mulheres com baixa ingestão de álcool. O consumo foi menor nesta população, pois esse hábito se destaca mais em homens, uma vez que, conforme a PNS ${ }^{21}$, o consumo abusivo de álcool foi superior entre os homens em comparação com as mulheres, com prevalências respectivas de $21,6 \%$ para os homens e $6,6 \%$ para as mulheres.

O consumo alimentar em associação com as DCNT não teve diferença significativa no estudo realizado, não sendo encontrados dados que possam comprovar que as mulheres possuíam práticas alimentares inadequadas. Esse dado é semelhante ao encontrado por Scherer et al. ${ }^{17}$, estudo no qual a maioria eram mulheres $(62,5 \%)$ com estado nutricional de sobrepeso. No caso dessas, destaca-se que consumiam diariamente leite integral e carnes, apresentavam consumo semanal de 1 a 3 vezes de frituras, e consumo de 1 a 2 vezes por semana de doces ${ }^{17}$.

Conforme os dados obtidos no presente estudo, verificou-se o consumo de alguns alimentos protetores, como $73,9 \%$ de ingestão diária de frutas frescas ou salada de frutas, consumo semanal de leguminosas para $77,0 \%$ e $76,4 \%$ de legumes e verduras cozidas. No entanto, se observados os demais dados encontrados, considerando o consumo de cereais, pão integral, centeio, nozes, castanhas, amendoim, leite desnatado, leite semidesnatado e iogurte light, os quais não faziam parte das práticas diárias de consumo alimentar da maioria das mulheres avaliadas, entende-se que é necessário monitoramento quanto à possibilidade de aumento da prevalência de DCNT nessa população.

Em comparação com a Pesquisa Nacional da Saúde ${ }^{6}$, as mulheres apresentaram consumo inferior de feijão se comparado aos homens, com proporções de $67,6 \%$ e $76,8 \%$, respectivamente, e $69,6 \%$ declaram beber leite integral. Em outro estudo, verificou-se que o consumo de leite integral e queijos amarelos (87\%) foi superior ao de leite desnatado e ao de queijo branco $(63 \%)^{22}$.

O consumo de carnes no presente estudo foi maior para carne de gado e peito de frango, enquanto o consumo de peixe mostra-se como uma prática mensal. Esses dados são semelhantes a outro estudo realizado, no qual o consumo de diferentes tipos de carnes apresentou alto índice pelos entrevistados, já o peixe foi quase esquecido pela população, com índices de consumo de $37 \% 22$. O baixo consumo relatado pela maioria das mulheres do presente estudo pode ser atribuído ao valor comercial do produto e à sua baixa disponibilidade no município, considerando que esse se localiza distante do mar.

Segundo estudo de Lins et al. ${ }^{23}$, a maioria das mulheres referiu ter uma alimentação saudável $(73,6 \%)$, hábito que aumenta positivamente de acordo com o aumento da renda, consumindo verduras e frutas regularmente com valores respectivamente de $80,2 \%$ e $54,1 \%$. No entanto $64,0 \%$ referiram não realizar uma alimentação saudável, por conseguinte constatou-se que estavam acima do peso, quando somadas as prevalências do excesso de peso e obesidade. Desse modo, entende-se que não realizar uma alimentação saudável está associado à obesidade ${ }^{23}$.

Dentre as limitações do presente estudo cita-se a dificuldade encontrada durante a coleta de dados quanto ao relato do questionário de frequência alimentar, no qual grande parte das mulheres ficava em dúvida ou não recordavam rapidamente a resposta para o consumo habitual de certos alimentos. Esse viés de memória, no entanto, é comum nos estudos transversais. Outro aspecto importante dos estudos com delineamento transversal é que estes não permitem a realização de associações do tipo causa e efeito. Além disso, esses dados não representam todo o munícipio de Guaporé-RS, mas apontam a necessidade de atenção à saúde das mulheres, pois, apesar das dificuldades e delineamento do estudo, este é relevante, na medida em que sinaliza que, entre as mulheres avaliadas, a prevalência de DCNT foi de aproximadamente $40 \%$ e que se associou com fatores de risco clássicos já descritos na literatura. Nessa perspectiva, faz-se necessário manter e intensificar medidas de promoção e prevenção pelos profissionais da saúde, uma vez que as políticas públicas e ações programáticas já estão definidas pelo Ministério da Saúde. Além disso, cabe lembrar que essas são as condições mais frequentes e que oneram o sistema de saúde. Nesse sentido, o monitoramento da prevalência das DCNT e ações para minimizá-las são importantes, a fim de garantir uma melhor qualidade de vida 
para a população e minimizar os custos na saúde pública da maioria dos municípios.

Neste estudo a prevalência de doenças crônicas associou-se com alguns dos fatores de risco estudados, entre esses: maior faixa etária, viúvas, menor escolaridade e renda, circunferência da cintura e índice de massa corporal.

\section{REFERÊNCIAS}

1. Brasil. Portal da Saúde. Vigilância das Doenças Crônicas Não Transmissíveis 2014 [Internet]. [capturado 2016 Ago 29]. Disponível em: http://portalsaude.saude.gov.br/index.php/o-ministerio/ principal/leia-mais-o-ministerio/671-secretaria-svs/vigilancia-de-a az/doenças-crônicas-nãotransmissiveis/14125-vigilancia-dasdoencas-cronicas-nao-transmissiveis

2. Brasil. Ministério da Saúde e Secretaria de Vigilância em Saúde. Departamento de Análise de Situação de Saúde, Plano de Ações. Estratégicas para o Enfrentamento das Doenças Crônicas Não Transmissíveis (DCNT) no Brasil 2011-2022, Brasília: Ministério da Saúde; 2011.

3. Villalpando S, Rodrigo JR. The status of non-transmissible chronic disease in Mexico based on the National Health and Nutrition Survey 2006. Introduction. Salud Publica Mex. 2010;52 Suppl 1: S2-3. https://doi.org/10.1590/S0036-36342010000700002

4. Goulart FAA, Kemper ES. Doenças crônicas não transmissíveis: Estratégias de controle e desafios para os sistemas de saúde. Brasília (DF): Organização Pan-Americana da Saúde/Organização Mundial da Saúde; 2011.

5. Brasil. Ministério da Saúde. Secretaria de Vigilância em Saúde. Departamento de Vigilância de Doenças e Agravos não Transmissíveis e Promoção da Saúde. Vigitel Brasil 2014: vigilância de fatores de risco e proteção para doenças crônicas por inquérito telefônico. Ministério da Saúde, Secretaria de Vigilância em Saúde, Departamento de Vigilância de Doenças e Agravos não Transmissíveis e Promoção da Saúde. Brasília: Ministério da Saúde; 2015.

6. Instituto de Pesquisa de Geografia e Estatística. Pesquisa Nacional de Saúde 2013 - Percepção do Estado de Saúde, Estilo de Vida e Doenças Crônicas. Rio de Janeiro: IBGE; 2014.

7. Matsudo SM, Araújo TL, Matsudo VKR, Andrade DR, Andrade EL, Oliveira LC Questionário Internacional de Atividade Física (IPAQ): estudo de validade e reprodutibilidade no Brasil. Rev Bras Ativ Saúde. 2001;10:5-18.

8. Rocha BSC, Dell Agnolo CM, França GAA, Romeiro LTC, Barros CMD. Doenças crônicas não transmissíveis e Associações com Fatores de Risco. Rev Bras Cardiol. 2014;27(1):35-42.

9. World Health Organization. Physical Status: The use and interpretation of anthropometry. Geneva: WHO; 1995.

10. World Health Organization, Obesity: preventing and managing the global epidemic: Report of a WHO consultation on obesity. Geneva; 2000 .
11. Atalah SE, Castillo LC, Castro SR, Aldea PA. The Nutrition Screening Initiative, Incorporating nutrition screening and interventions into medical practice: a monograph for physicians. Washington D.C.: American Academy of Family Physicians; 1994.

12. Mannato LW. Questionário de Frequência alimentar ELSA BRASIL: proposta de redução e validação reduzida. Vitória: UFES; 2013.

13. Neumann B, Conde SR, Lemos JRN, Moreira TR. Associação entre estado nutricional e a prevalência de doenças crônicas não transmissíveis em idosos residentes no município de Roca Sales RS. Rev Bras Ciênc Env Human. 2014:11(2):166-77.

14. Cavalcanti CL, Gonçalves RMC, Asciutti LSR, Cavalcanti AL. Prevalência de doenças crônicas e estado nutricional em um grupo de idosos brasileiros, Rev Salud Pública. 2009;11(6):865-77. https:// doi.org/10.1590/S0124-00642009000600003

15. Kim HC, Oh SM. Noncommunicable Diseases: Current Status of Major Modifiable Risk Factors in Korea. J Prev Med Public Health. 2013;46(4):165-72. https://doi.org/10.3961/jpmph.2013.46.4.165

16. Oliveira MJI, Espirito SE. A relação entre os determinantes sociais da saúde e a questão social. Cad Saúde Desenvol. 2013; 2(2):8-24.

17. Scherer R, Scherer F, Rufatto CS, Dal Bosco SM. Estado nutricional e prevalência de doenças crônicas em idosos de um município do interior do Rio Grande do Sul. Rev Bras Geriat. 2013; 16(4):769-79. https://doi.org/10.1590/S1809-98232013000400011

18. Bortoletto MSS, Souza RTK, Cabrera MAS, González AD. Síndrome metabólica, componentes e fatores associados em adultos de 40 anos ou mais de um munícipio da Região Sul do Brasil. Cad Saúde Colet. 2016;24(1):32-40. https://doi.org/10.1590/1414462X201600010123

19. Santos Alves JW, Soares NT, Diniz NA, Santos PED, Monteiro RS. Estado nutricional, estilo de vida, perfil socioeconômico e consumo alimentar de comerciários. Rev Bras Prom Saud. 2012;25(2 Supl):40-50. https://doi.org/10.5020/18061230.2012.s40

20. Malta DC, Araújo Andrade SSC, Stopa SR, Pereira CA, Szwarcwald $\mathrm{CL}$, Silva Júnior JB, Reis AAC. Estilo de vida da população brasileira: resultados da Pesquisa Nacional de Saúde, 2013. Epidemiol Serv Saud. 2015;24(2):217-26. https://doi.org/10.5123/S167949742015000200004

21. Freitas SLR, Garcia LP. Consumo abusivo de álcool no Brasil: resultados da Pesquisa Nacional de Saúde. Epidemiol Serv Saud. 2013;24(2):227-37. http://dx.doi.org/10.5123/S167949742015000200005

22. Orlando R, Pinheiro TLF, Volkweis DSH, Colussi EL. Avaliação da alimentação e sua relação com as doenças crônicas não transmissíveis de um grupo de idosos de um município da região norte do estado do RS. Rev Enferm. 2011;6(6-7):203-17.

23. Lins APM, Schieri R, Coutinho WF, Ramos EG, Peixoto MVM, Fonseca VM. Alimentação saudável, escolaridade e excesso de peso entre mulheres de baixa renda. Ciênc Saúde Colet, 2013;18(2): 357-66. https://doi.org/10.1590/S1413-81232013000200007 